\title{
Prevention of Maternal and Congenital Cytomegalovirus Infection
}

\author{
Julie Johnson, MD1 , Brenna Anderson, MD, MSc ${ }^{2}$, and Robert F. Pass, MD ${ }^{3}$ \\ ${ }^{1}$ Department of Obstetrics and Gynecology, Eastern Virginia School of Medicine, Norfolk, VA \\ ${ }^{2}$ Department of Obstetrics and Gynecology, Women and Infants Hospital/Alpert Medical School of \\ Brown University, Providence, RI \\ ${ }^{3}$ Department of Pediatrics, University of Alabama at Birmingham, Birmingham, AL
}

\begin{abstract}
Congenital cytomegalovirus (CMV) infection is an important cause of hearing impairment, mental retardation and cerebral palsy. Principal sources of infection during pregnancy are young children and intimate contacts. Prevention of maternal and congenital CMV infection depends on counseling women regarding the sources of infection and hygienic measures that might prevent infection. There is currently insufficient evidence to support use of antiviral treatment or passive immunization for post-exposure prophylaxis of pregnant women or as a maternal treatment aimed at preventing fetal infection. Vaccines for CMV are under development but it will be a number of years before one is licensed.
\end{abstract}

\section{Keywords}

Cytomegalovirus; congenital infection; CMV prevention; prenatal counseling

\section{INTRODUCTION}

Cytomegalovirus (CMV) is one of the most common causes of congenital infection, with an overall prevalence of approximately $0.64 \%$ of all births. ${ }^{1,2}$ The prevalence varies according to geography, socioeconomic status and ethnicity. ${ }^{3}$ The average birth prevalence in the United States is approximately $0.5-1 \%$, which translates into approximately 40,000 cases annually. ${ }^{1,4}$ Congenital CMV affects more children than other more commonly known conditions such as Down syndrome, fetal alcohol syndrome, and spina bifida. ${ }^{5}$ Almost 400 children die each year from this disease, and up to 8000 develop permanent disabilities such as developmental delay and hearing or vision loss. ${ }^{6,7}$ With an estimated cost of $\$ 300,000$ per infected child, the annual costs associated with this disease burden approach \$1-\$2 billion. ${ }^{7}$ Given the societal and economic burden of this disease, a comprehensive, national program for prevention of $\mathrm{CMV}$ infection in pregnant women is needed. Obstetricians can play a significant role in this effort by being knowledgeable about the natural history of maternal and congenital CMV infection, by being prepared to provide prevention

Correspondence: Robert F. Pass, MD, Children's Hospital Suite 108, $16007^{\text {th }}$ Avenue South, Birmingham, AL 35233, Voice: 205-939-9922, Fax: 205-934-7273, rpass@ peds.uab.edu.

Reprints will not be available.

Conflicts of Interest and Source of Funding: JJ has nothing to declare. BA receives research funding from the Eunice Kennedy Shriver National Institute of Child Health and Human Development (NICHD) K23 HD062340-01 and has no conflicts to declare. RFP has received funding from the National Institutes of Allergy, Immunology and Infectious Diseases (U01-AI063565) and from Sanofi Pasteur and is a consultant to Astellas, Merck and the State of Montana Attorney General's office. 
information to their patients and by seeing that newborns with suspected or proven congenital CMV infection are referred to pediatric specialists for evaluation and management.

\section{BIOLOGY OF HUMAN CMV INFECTION}

\section{The Virus}

Human cytomegalovirus, a member of the herpesvirus family, is a large enveloped virus that is widely distributed in human populations. Its double stranded DNA includes approximately 160 open reading frames. A large portion of the human CMV genome encodes gene products that evade or interfere with host immune responses or interact with inflammatory molecules in ways that facilitate persistence and dissemination of CMV in the host. $^{8}$

\section{Primary CMV Infection}

Initial infection with $\mathrm{CMV}$ can produce a heterophile negative mononucleosis characterized by prolonged fever (>2 weeks), malaise, headache, pharyngitis, lymphadenopathy, hepatosplenomegaly and arthralgias that cannot be distinguished clinically from mononucleosis due to Epstein-Barr virus. ${ }^{8}$ However, primary CMV infection is almost always clinically silent in healthy children and adults, including pregnant women. ${ }^{4}$ Infection is thought to be initiated by inoculation of virus onto a mucosal site by hand contact or intimate contact. The incubation period, the time from inoculation to onset of viral excretion or symptoms, is around one month. With primary CMV infection, viremia is present and virus is shed from multiple sites (saliva, urine, cervicovaginal secretions and semen). Antibody (IgM and IgG) to CMV appears in serum soon after onset of viral shedding; envelope glycoproteins are the target of neutralizing antibodies. Antibody avidity is low during the first few months after CMV infection but increases thereafter; low antibody avidity has been used diagnostically to confirm recent onset of CMV infection in pregnant women. ${ }^{9} \mathrm{CD} 8$ cytotoxic T lymphocyte responses appear at about the same time and are a prominent part of the host immune response to CMV, targeting multiple viral proteins, notably matrix and regulatory proteins. ${ }^{10,11} \mathrm{CD} 4 \mathrm{~T}$ lymphocyte responses develop more slowly over the following months. Viremia continues for weeks to months, even in the presence of antibody and T-lymphocyte responses to CMV. ${ }^{12}$ Shedding of CMV in body fluids continues for months to more than a year in adults and may continue for several years in young children, especially when infection occurred during the first year of life.

\section{Latency, Reactivation and Reinfection}

CMV persists in the human host indefinitely after primary infection. Similar to other members of the herpesvirus family, CMV becomes latent reactivating to active viral replication after stimuli such as inflammation or immune impairment due to disease or medical treatment. Hormonal changes associated with pregnancy and lactation may also stimulate reactivation of CMV. Shedding of CMV in urine and cervico-vaginal fluids increases during pregnancy with increasing gestational age and is also increased during the first few months postpartum. ${ }^{3,13}$ In addition, CMV is commonly shed in human milk from two to 12 weeks postpartum; over $90 \%$ of CMV-seropositive women have milk that is positive by PCR. ${ }^{14}$

Considering the difficulties presented by CMV infections in immunocompromised hosts and the generally benign nature of CMV infection in the normal host, one could conclude that the immune response is largely able to control the virus. However, the immune response is not able to stop viral replication during the first months of infection, nor can it completely prevent intermittent viral shedding or reinfection. Reinfection in normal hosts with past 
CMV infection was demonstrated in the past using DNA fingerprinting of viral DNA to demonstrate acquisition of a new, genetically distinct virus. More recently studies in CMV immune women who gave birth to babies with congenital CMV infection found they had acquired a virus that contained an epitope on an envelope glycoprotein that was not recognized by antibodies generated by the past infection. ${ }^{15}$ It appears that congenital CMV infection in babies born to CMV immune mothers may be due more often to reinfection than to reactivation of endogenous, latent virus.

\section{EPIDEMIOLOGY}

Seroprevalence rates in women of reproductive age range from $40-83 \%$, with women of lower socioeconomic status having a higher rate of previous infection. ${ }^{16,17}$ Seroconversion, representing a primary infection during pregnancy, occurs in approximately $1-4 \%$ of seronegative, pregnant women. $3,7,18$ Recent risk estimates for acquiring a primary infection during pregnancy based on risk estimates derived from CMV prevalence data are $1.38 \%$, $3.4 \%$, and $3.85 \%$ among non-Hispanic whites, non-Hispanic blacks, and Mexican Americans, respectively. ${ }^{19}$ This translates into approximately 27,000 primary infections during pregnancy per year. Congenital infection occurs through primary maternal infection, reactivation of a latent infection, or reinfection with a new strain of the virus. The latter two - reactivation and reinfection - are often referred to as recurrent infection or nonprimary infection because aside from a relatively small number of cases that have been the subject of careful laboratory investigation, it is usually not possible to know whether a nonprimary maternal infection was due to reactivation or reinfection. In many populations the majority of congenital CMV infections are the result of recurrent (nonprimary) maternal infection; this is particularly true in developing countries and in low income populations in developed countries. However, the risk of having a newborn with congenital CMV infection is higher for a woman who is seronegative than one who has had CMV infection in the past. ${ }^{20} \mathrm{In}$ addition, primary maternal CMV infection is more likely to result in congenital CMV with disabilities than is recurrent maternal infection. ${ }^{21}$

\section{Sources of Maternal Infection}

Transmission of CMV appears to require direct contact with body fluids from a person who is shedding CMV. The source of CMV infection for a young woman is likely to be someone close to her such as a preschool age child, a spouse or sexual contact. The incidence of CMV infection in healthy, susceptible (seronegative) young adults in the U.S. is approximately $2 \%$ per year based on studies in blood donors and hospital personnel. Table 1 lists situations in which increased rates of CMV infection have been observed. Rates of infection among day care workers, parents of children who are shedding CMV and sexually active adolescents are several-fold greater than the background rate of around $2 \%$ per year. Rates of infection of near 50\% per year have been reported in parents whose child of less than two years was shedding CMV. The quantity of virus in body fluids decreases over time after primary CMV infection and person to person spread through intimate contact is more likely if the source has recently acquired CMV. Contact with young children who have CMV infection is associated with high risk of infection because these children have been infected relatively recently, they shed virus in body fluids for years and their care typically involves contact with urine, saliva and other body fluids.

\section{DIAGNOSIS OF MATERNAL, FETAL AND CONGENITAL INFECTION}

\section{Antibody tests}

Detection of serum $\operatorname{IgG}$ antibody indicates that CMV infection occurred in the recent or distant past. The value of testing for $\mathrm{IgG}$ antibody is to determine whether a patient (or blood or organ donor) has ever been infected by CMV. Serial testing in a patient who is 
initially CMV IgG antibody negative can be used to prove primary infection; conversion from CMV IgG antibody negative to positive confirms primary infection. The amount of $\mathrm{IgG}$ antibody to CMV cannot be used to accurately predict recent infection or CMV disease. IgM antibody is often used to help determine whether a patient has a recent CMV infection. However, it is important to know that there is patient to patient variation in how long CMV IgM antibody persists after primary infection and there is a wide range in the ability of various assays to detect IgM antibody. With some assays, most subjects with primary CMV infection clear IgM antibody within two months and with other assays IgM antibody can be detected for 6 to 9 months. To make matters more complicated, some people with CMV infection in the distant past may have IgM antibody to CMV during pregnancy or after immunosuppression. Antibody avidity reflects the strength of binding between a polyvalent antigen and antibody. Antibody avidity increases with time after initial infection and low $\mathrm{IgG}$ antibody avidity has been used to identify subjects with recent primary infection by CMV and other pathogens. Evaluation of a pregnant woman suspected of having a primary CMV infection should include initial testing for both CMV IgG and IgM antibodies. If the subject is CMV IgG antibody negative, testing should be repeated in two to four weeks regardless of the initial IgM result. More often than not, detection of CMV IgM antibody in the absence of $\operatorname{IgG}$ antibody is due to a false positive IgM antibody test. If the subject is IgG and IgM antibody positive on the first or second test, testing for CMV IgG antibody avidity can help sort out whether the infection has occurred in recent months or in the more distant past. If the subject is positive only for IgG antibody but primary CMV infection is strongly suspected, CMV IgG antibody avidity testing could provide useful information on the timing of the infection. Using a step-wise approach that included testing for IgG antibody, enzyme immunoassay and immunoblot tests for IgM antibody and CMV IgG antibody avidity in a research laboratory, pregnant women with primary CMV infection were accurately identified from among those referred for evaluation. ${ }^{9}$ Whether the assays available commercially to the practicing physician in the U.S. will work as well is not known. Unfortunately, there is considerable variation between commercially available assays in sensitivity and specificity for detection of IgM antibody, persistence of IgM antibody after primary infection and accuracy of "low" CMV IgG antibody avidity for prediction of recent infection. ${ }^{22}$ It is important to consult with the laboratory that provides testing in order to learn as much as possible about the reliability of the tests offered for identification of recent primary CMV infection.

The above discussion of antibody testing is aimed at evaluation of the pregnant woman suspected of having primary CMV infection because of a mononucleosis-like illness, exposure to someone with CMV infection, occupational exposure (day care worker), a positive result from a screening test for CMV antibody or a fetal ultrasound suggestive of congenital infection. In these situations, the pretest probability of primary CMV infection is relatively high. The use of antibody tests to screen pregnant women for CMV infection is an entirely different matter. The pretest probability of a true positive result is much smaller when screening all prenatal patients and tests with very high specificity are required in order to avoid a preponderance of false positive screens which result in unnecessary psychological stress to the mother, expensive and invasive evaluations and the possibility of needless terminations. Screening pregnant women for CMV infection remains a controversial issue primarily because of uncertainty over the availability of laboratory tests with the needed level of sensitivity and specificity and the limited options for intervention if primary CMV infection is identified.

\section{Detection of Maternal CMV Excretion or Viremia}

Detection of CMV is not routinely used in the evaluation of a pregnant woman for primary infection. Shedding of CMV in urine, saliva or vagina and viremia could occur even if 
infection was not recent. Detection of virus in the amniotic fluid or in newborn urine or saliva is the definitive test for fetal and neonatal infection respectively. For diagnosis of fetal (amniotic fluid sample) and congenital CMV infection (urine or saliva sample), standard viral culture and polymerase chain reaction (PCR) have similar sensitivity.

\section{Detection of Fetal Infection}

If primary maternal infection is confirmed or if fetal ultrasound suggests congenital infection and maternal CMV infection cannot be ruled out, an attempt at virologic diagnosis of fetal infection can be considered. However, it is important to discuss the risks and benefits of this endeavor with the mother; this discussion should include information on the probability that fetal infection will be detected and the probability of CMV related disability if fetal infection is proven. Patients who would not consider termination of pregnancy regardless of fetal condition or CMV diagnosis usually do not choose to undergo prenatal diagnosis. The fetus excretes CMV in urine, so the renal system should be functional to detect the virus in amniotic fluid. In addition transmission of virus from mother to fetus may not occur shortly after maternal infection. For these reasons, amniocentesis should be reserved until approximately 20-21 weeks gestation and 6-8 weeks after maternal infection in order to avoid false negative results. ${ }^{23} \mathrm{PCR}$ is preferred for prenatal diagnosis of fetal infection because the result can be available in one day; CMV isolation in tissue culture has similar sensitivity and better specificity for detection of virus in amniotic fluid, but it can take two weeks or longer to provide a result.

Ultrasound may also be used as an adjunct to amniocentesis. Some markers associated with fetal CMV infection are cerebral ventriculomegaly, microcephaly, echogenic bowel, hepatosplenomegaly, intracranial or intra-abdominal calcifications, growth restriction, and placental enlargement. ${ }^{24,25}$ However, lack of ultrasound findings does not exclude congenital infection; up to $47 \%$ of fetuses with congenital CMV infection may have normal ultrasound findings and if the diagnosis of fetal CMV infection has not been established virologically, ultrasound is a relatively poor predictor of symptomatic congenital infection.

\section{Diagnosis of Congenital Infection}

Whether or not prenatal diagnosis was attempted, newbornsborn to a mother with primary CMV infection during pregnancy should be tested for congenital infection. Detection of CMV in the body fluids of the newborn within the first three weeks of life by PCR or viral culture establishes the diagnosis of congenital infection. Urine or saliva are the preferred samples to test. After three weeks of age it is possible that CMV shedding by the newborn could be the result of intrapartum or breast milk acquired infection. Newborns who are symptomatic will be candidates for ganciclovir or valganciclovir treatment. All infants with congenital CMV infection should be evaluated carefully for hearing loss, retinitis and neurological function and their follow-up should include serial assessment of hearing function and development.

\section{PREVENTION OF MATERNAL AND CONGENITAL CMV INFECTION}

\section{Preventing Maternal Exposure}

In the absence of a licensed vaccine, prevention of CMV infection presents challenges due to the biology and epidemiology of human infection. Although situations with increased risk are known, it is very unlikely that exposure will be linked to contact with a specific person because persons shedding CMV are unlikely to have any illness. Even if contact with someone known to have recent CMV infection has occurred, it is possible (even likely in the case of childcare or hospital workers) that there has been contact with other persons shedding CMV. There is currently no role for post-exposure prophylaxis with antivirals or 
immune globulin. The only means of preventing maternal CMV infection that is available at this time is by limiting exposure to the virus. Efforts at limiting exposure are based on hygiene, with the focus on hand hygiene in settings where women who are or could become pregnant have contact with young children. This includes women who work in childcare and mothers of young children. Specific recommendations for preventing transmission of CMV have been made by the Centers for Disease Control and Prevention (CDC); these are summarized in Table 2. 26,27

The fact that CMV infection rates are not increased among hospital workers who care for children suggests that standard precautions aimed at preventing contact with body fluids of patients are effective in preventing transmission of CMV in the hospital setting. Whether this approach can be adapted for widespread use in the home where the source of maternal infection will be a spouse or young child with whom there is daily close contact has not been determined. However, results of two studies that provided specific instructions to CMV seronegative pregnant women on CMV infection and steps to prevent CMV infection by avoiding contact with body fluids from others suggest that this approach to prevention can be effective outside the hospital setting. ${ }^{28,} 29$

\section{Vaccine}

An effective vaccine for prevention of maternal CMV infection is needed, given the difficulties in preventing transmission of a virus that is shed in body fluids of asymptomatic, healthy children and adults for months to years and the fact that the source of a maternal infection that leads to fetal infection will often be contact with a family member. The Institute of Medicine of the National Academy of Sciences has identified prevention of congenital CMV infection as a top priority for vaccine development for the U.S. for the $21^{\text {st }}$ century based on an analysis of cost-effectiveness and quality of life ${ }^{30}$ Interest in CMV vaccine development is growing with several major vaccine companies conducting phase I and II clinical trials with investigational vaccines. ${ }^{31}$ A randomized, placebo controlled phase II clinical trial of a subunit vaccine comprised of recombinant CMV envelope glycoprotein B and MF59 adjuvant in young mothers demonstrated 50\% efficacy for prevention of infection over a three year period. ${ }^{32}$ However, no CMV vaccine is currently licensed and no phase III clinical trials are underway so it will be a number of years before a vaccine is available.

\section{Preventing Transmission of CMV from Mother to Fetus}

In a study of 181 women with CMV infection during pregnancy, the effect of passive immunization with a CMV immune globulin preparation on the outcome of fetal infection and the risk of transmission of CMV to the fetus were studied. ${ }^{33}$ Among 55 pregnancies in which fetal infection was diagnosed by detection of virus in amniotic fluid, 31 subjects accepted treatment with CMV immune globulin, 14 declined and 10 chose to have an abortion. The number of newborns with symptomatic congenital CMV disease was $1(3 \%)$ in the treatment group and 7 (50\%) in the untreated subjects who carried newborns to term. Although this result suggests a dramatic effect of CMV immune globulin in treating fetal CMV disease, the fact that the treatment assignment was not randomized and observers were not blinded raises a significant concern about bias. In addition, 7 of the "infected" fetuses were CMV negative as newborns. Since there is no evidence that antibody to CMV has ever cleared the infection in a human host, this result calls into question the accuracy of the diagnosis of fetal infection in this study. The prevention group was comprised of women who did not undergo amniocentesis. Congenital CMV infection was detected in 6/37 (16\%) of newborns of mothers who received CMV immune globulin and in 19/47 (40\%) in the untreated group, a difference that was statistically significant. However, due to the small sample size, lack of randomization, heterogeneity of the prevention group in this study and 
the fact the fetal infection rate in the treatment group is close to that reported in subjects with first trimester CMV infection ${ }^{34}$, the efficacy of maternal CMV immune globulin for prevention of fetal CMV infection is uncertain. Two multi-site, randomized, placebo controlled clinical trials of CMV hyperimmune globulin treatment of pregnant women for prevention of congenital CMV infection should answer questions concerning the efficacy of this intervention (NCT00881517 and NCT01376778, both accessed at ClinicalTrials.gov, October 31, 2011). A clinical trial conducted in Italy is fully enrolled and has an anticipated completion date of March 2010. A clinical trial in the U.S. sponsored by the Eunice Kennedy Shriver National Institute of Child Health and Human Development will begin enrollment soon.

\section{Antiviral Treatment of Fetal Infection}

A study of pregnancies complicated by fetal CMV infection reported that valacyclovir administered orally at 28 weeks achieved therapeutic levels in amniotic fluid. ${ }^{35}$ However the outcome for the newborn did not appear to be different in babies born to treated mothers compared with a no treatment group. Ganciclovir, a more potent antiviral for CMV infection, is now used to treat symptomatic congenital CMV infection and is used widely to treat or prevent CMV infections in immunocompromised hosts. ${ }^{36} \mathrm{~A}$ case report described clearance of the virus from the amniotic fluid of an affected pregnancy and subsequent delivery of a healthy neonate with the use of prenatal ganciclovir. ${ }^{37}$ Although a randomized, controlled clinical trial with ganciclovir in pregnancies complicated by fetal infection could be very valuable, the potential toxicity of the drug and the wide variation in severity of disease would make such a trial challenging. Neither valacyclovir nor ganciclovir are approved for treatment of CMV infection during pregnancy at this time and there is insufficient data on efficacy of use during pregnancy to recommend these antivirals for prevention of transmission from mother to fetus or treatment of fetal infection.

\section{RECOMMENDATIONS FOR OBSTETRICIANS}

Obstetricians can incorporate assessment of maternal risk of CMV infection into routine prenatal care and provide counseling on prevention of infection. The American College of Obstetricians and Gynecologists (ACOG) recommends against routine serologic screening pregnant women for CMV infection. ${ }^{38}$ However, ACOG does recommend counseling patients at risk on methods for prevention of CMV infection, stating that “...women with young children or those who work with young children should be advised that the risk of infection can be reduced significantly by safe-handling techniques, such as the use of latex gloves and rigorous hand-washing after handling diapers or after exposure to respiratory secretions." 38 The Society of Obstetricians and Gynaecologists of Canada (GOGC), does not recommend routine prenatal serologic screening for CMV infection, but does make a number of recommendations for management of CMV infection during pregnancy, including serologic testing for CMV in women who develop an influenza-like illness or have sonographic findings suggestive of fetal CMV infection. ${ }^{39}$ Although the SOGC recommendations do not mention assessment of risk of CMV infection or specific methods of prevention for widespread use, they do recommend serologic monitoring during pregnancy for seronegative women at risk because of employment in health care or child care and for those who have a young child at home. ${ }^{39}$ Recommendations for prevention of CMV infection in pregnancy by the Centers for Disease Control and Prevention are summarized in Table 2 and are aimed at patients; no specific recommendations for action by obstetricians are made. ${ }^{26}$

In summary, it appears that currently the best option for prevention of congenital CMV is through education of patients regarding sources of maternal infection and methods of hygiene aimed at preventing maternal exposure to CMV. In a recent survey of obstetricians, 
only $60 \%$ routinely discuss hand washing with pregnant women. It is quite possible that routine assessment of CMV risk and provision of counseling regarding prevention by all obstetricians could significantly reduce the rate of congenital CMV infection.

\section{References}

1. Kenneson A, Cannon MJ. Review and meta-analysis of the epidemiology of congenital cytomegalovirus (CMV) infection. Rev Med Virol. 2007; 17:253-76. [PubMed: 17579921]

2. Dollard SC, Grosse SD, Ross DS. New estimates of the prevalence of neurological and sensory sequelae and mortality associated with congenital cytomegalovirus infection. Rev Med Virol. 2007; 17:355-63. [PubMed: 17542052]

3. Stagno S, Pass RF, Dworsky ME, et al. Maternal cytomegalovirus infection and perinatal transmission. Clin Obstet Gynecol. 1982; 25:563-76. [PubMed: 6290121]

4. Stagno, S.; Britt, W. Cytomegalovirus Infections. In: Remington, JS.; Klein, JO.; Wilson, CB.; Baker, CJ., editors. Infectious Diseases of the Fetus and Newborn Infant. 6. Philadelphia, PA: Elsevier Saunders; 2006. p. 739-81.

5. Ross DS, Dollard SC, Victor M, et al. The epidemiology and prevention of congenital cytomegalovirus infection and disease: activities of the Centers for Disease Control and Prevention Workgroup. J Womens Health (Larchmt). 2006; 15:224-9. [PubMed: 16620180]

6. Arvin AM, Fast P, Myers M, et al. Vaccine development to prevent cytomegalovirus disease: report from the National Vaccine Advisory Committee. Clin Infect Dis. 2004; 39:233-9. [PubMed: 15307033]

7. Cannon MJ, Davis KF. Washing our hands of the congenital cytomegalovirus disease epidemic. BMC Public Health. 2005; 5:70. [PubMed: 15967030]

8. Mocarski, ESJ.; Shenk, T.; Pass, RF. Cytomegaloviruses. In: Knipe, DM.; Howley, PM., editors. Fields Virology. 5. Philadelphia: Lippincott, Williams \& Wilkins; 2007. p. 2701-72.

9. Lazzarotto T, Varani S, Guerra B, et al. Prenatal indicators of congenital cytomegalovirus infection. J Pediatr. 2000; 137:90-5. [PubMed: 10891828]

10. Khan N, Cobbold M, Keenan R, et al. Comparative analysis of CD8+ T cell responses against human cytomegalovirus proteins pp65 and immediate early 1 shows similarities in precursor frequency, oligoclonality, and phenotype. J Infect Dis. 2002; 185:1025-34. [PubMed: 11930311]

11. Elkington R, Walker S, Crough T, et al. Ex vivo profiling of CD8+-T-cell responses to human cytomegalovirus reveals broad and multispecific reactivities in healthy virus carriers. J Virol. 2003; 77:5226-440. [PubMed: 12692225]

12. Revello MG, Zavattoni M, Sarasini A, et al. Human cytomegalovirus in blood of immunocompetent persons during primary infection: prognostic implications for pregnancy. $\mathrm{J}$ Infect Dis. 1998; 177:1170-5. [PubMed: 9592999]

13. Pass RF, Stagno S, Dworsky ME, et al. Excretion of cytomegalovirus in mothers: observation after delivery of congenitally infected and normal infants. J Infect Dis. 1982; 146:1-6. [PubMed: 6282987]

14. Schleiss MR. Role of breast milk in acquisition of cytomegalovirus infection: recent advances. Curr Opin Pediatr. 2006; 18:48-52. [PubMed: 16470162]

15. Boppana SB, Rivera LB, Fowler KB, et al. Intrauterine transmission of cytomegalovirus to infants of women with preconceptional immunity. N Engl J Med. 2001; 344:1366-71. [PubMed: 11333993]

16. Gaytant MA, Steegers EAP, Semmekrot BA, et al. Congenital cytomegalovirus infection: review of the epidemiology and outcome. Obstet Gynecol Surv. 2002; 57:245-56. [PubMed: 11961482]

17. Staras SAS, Dollard SC, Radford K, et al. Cytomegalovirus infection in the United States: seroprevalence and demographic risk factors. Clin Infect Dis. 2006; 43:1143-51. [PubMed: 17029132]

18. Ludwig A, Henge H. Epidemiological impact and disease burden of congenital cytomegalovirus infection in Europe. Eurosurveillance. 2009; 14:1-7. 
19. Colugnati FAF, Staras SAS, Dollard SC, et al. Incidence of cytomegalovirus infection among the general population and pregnant women in the United States. BMC Infectious Diseases. 2007; 7:71. [PubMed: 17605813]

20. Fowler KB, Stagno S, Pass RF. Maternal immunity and prevention of congenital cytomegalovirus infection. JAMA. 2003; 289:1008-11. [PubMed: 12597753]

21. Fowler KB, Stagno S, Pass RF, et al. The outcome of congenital cytomegalovirus infection in relation to maternal antibody status. N Engl J Med. 1992; 326:663-7. [PubMed: 1310525]

22. Revello MG, Genini E, Gorini G, et al. Comparative evaluation of eight commercial human cytomegalovirus IgG avidity assays. J Clin Virol. 2010; 48:255-9. [PubMed: 20561816]

23. Liesnard C, Donner C, Brancart F, et al. Prenatal diagnosis of congenital cytomegalovirus infection: prospective study of 237 pregnancies at risk. Obstetrics and Gynecology. 2000; 95:8818. [PubMed: 10831985]

24. Guerra B, Simonazzi G, Puccetti C, et al. Ultrasound prediction of symptomatic congenital cytomegalovirus infection. Am J Obstet Gynecol. 2008; 198:380.e1-e-7. [PubMed: 18191802]

25. Benoist G, Salomon LJ, Jacquemard F, et al. The prognositc value of ultrasound abnormalities and biological parameters in blood of fetuses infected with cytomegalovirus. BJOG. 2008; 115:823-9. [PubMed: 18485159]

26. [Accessed March 15, 2011] Preventing Congenital CMV Infection. 2011. at http://www.cdc.gov/cmv/prevention.html

27. [Accessed March 15, 2011] People who care for infants and children. 2011. at http://www.cdc.gov/cmv/risk/infants-children.html

28. Adler SP, Finney JW, Manganello AM, et al. Prevention of child-to-mother transmission of cytomegalovirus among pregnant women. J Pediatr. 2004; 145:485-91. [PubMed: 15480372]

29. Velloup-Fellous C, Picone O, Cordier A-G, et al. Does hygiene counseling have an impact on the rate of CMV primary infection during pregnancy? Results of a 3-year prospective study in a French hospital. J Clin Virol. 2009; 46S:S49-S53.

30. Stratton, K.; Durch, J.; Lawrence, R. Vaccines for the 21st Century: A Tool for Decisionmaking. Washington, DC: National Academy Press; 2001.

31. Schleiss MR. Cytomegalovirus vaccine development. Curr Top Microbiol Immunol. 2008; 325:361-82. [PubMed: 18637516]

32. Pass RF, Zhang C, Evans A, et al. Vaccine prevention of maternal cytomegalovirus infection. $\mathrm{N}$ Engl J Med. 2009; 360:1191-9. [PubMed: 19297572]

33. Nigro G, Adler SP, La Torre R, et al. Passive immunization during pregnancy for congenital cytomegalovirus infection. N Engl J Med. 2005; 353:1350-62. [PubMed: 16192480]

34. Griffiths PD, Baboonian C. A prospective study of primary cytomegalovirus infection during pregnancy: final report. Br J Obstet Gynaecol. 1984; 91:307-15. [PubMed: 6324849]

35. Jacquemard F, Yamamoto M, Costa J-M, et al. Maternal administration of valaciclovir in symptomatic intrauterine cytomegalovirus infection. BJOG. 2007

36. Kimberlin DW, Lin CY, Sanchez PJ, et al. Effect of ganciclovir therapy on hearing in symptomatic congenital cytomegalovirus disease involving the central nervous system: a randomized, controlled trial. J Pediatr. 2003; 143:16-25. [PubMed: 12915819]

37. Puliyanda DP, Silverman NS, Lehman D, et al. Successful use of oral ganciclovir for the treatment of intrauterine cytomegalovirus infection in a renal allograft recipient. Transpl Infect Dis. 2005; 7:71-4. [PubMed: 16150094]

38. ACOG. Clinical management guidelines for obstetrician-gynecologists, perinatal viral and parasitic infections. Washington, D.C: 2000.

39. SOGC. Cytomegalovirus infections in pregnancy. J Obstet Gynaecol Can. 2010; 240:348-54. 


\section{Table 1}

Rates of CMV infection in various groups. (Adapted from Field's Virology, $5^{\text {th }}$ Edition, p. $2737^{8}$ with permission).

\begin{tabular}{|lc|}
\hline Group & Incidence rate, percent/year \\
\hline Blood donors & 1.57 \\
Hospital employees & $2-2.2$ \\
Pregnant women, low income & 6.8 \\
Pregnant women, middle income & 2.5 \\
Women in STD clinic & 37 \\
Adolescent females & 13.8 \\
Parents of CMV shedding child, aged 0-12 months & 47 \\
Parents of CMV shedding child $\leq 18$ months of age & 32 \\
Parents of CMV shedding child 19 months to 6 years of age & 13 \\
Daycare workers & $7.9-20$ \\
\hline
\end{tabular}


Table 2

Steps recommended by the Centers for Disease Control and Prevention for reducing CMV exposure in pregnant women and thus reducing the risk of fetal infection.

- Wash your hands often with soap and water for 15-20 seconds, especially after

- $\quad$ changing diapers

- $\quad$ feeding a young child

- $\quad$ wiping a young child's nose or drool

- $\quad$ handling children's toys

- Do not share food, drinks, or eating utensils used by young children

- Do not put a child's pacifier in your mouth

- Do not share a toothbrush with a young child

- Avoid contact with saliva when kissing a child

- Clean toys, countertops, and other surfaces that come into contact with children's urine or saliva 